\title{
Correlative Microscopy based on Secondary Ion Mass Spectrometry for High- Resolution High-Sensitivity Nano-Analytics
}

\author{
T. Wirtz, D. Dowsett, S. Eswara Moorthy
}

Advanced Instrumentation for Ion Nano-Analytics (AINA), Luxembourg Institute of Science and Technology, 41 rue du Brill, L-4422 Belvaux, Luxembourg

Nano-analytical techniques and instruments providing both excellent spatial resolution and highsensitivity chemical information are of extreme importance in materials science and life sciences for investigations at the nanoscale. Due to the ever increasing complexity of devices and the continuously shrinking geometries in materials research, characterization tools and techniques are facing new challenges and need to anticipate future trends.

Electron Microscopy, Helium Ion Microscopy and Scanning Probe Microscopy are commonly used for high-resolution imaging. However, these techniques all have the same important drawback: they provide no or only very limited chemical information. In electron microscopy, chemical information can be obtained by using techniques like EELS or EDS, but the sensitivity is limited. Moreover, these techniques do not permit to distinguish between isotopes, which is a major handicap today due to the increasing use of isotopic labeling, and have limitations in the low mass range. By contrast, Secondary Ion Mass Spectrometry (SIMS) is an extremely powerful technique for analyzing surfaces owing in particular to its excellent sensitivity, high dynamic range, very high mass resolution and ability to differentiate between isotopes.

In order to get chemical information with a highest sensitivity and highest lateral resolution, we have investigated the feasibility of combining SIMS with Transmission Electron Microscopy, Scanning Probe Microscopy and Helium Ion Microscopy and developed three prototype instruments corresponding to these three combinations:

- TEM - SIMS : FEI Tecnai F20 equipped with a $\mathrm{Ga}^{+}$FIB column and dedicated SIMS extraction optics, mass spectrometer and detectors (figure 1)

- HIM - SIMS : Zeiss ORION Helium Ion Microscope with dedicated SIMS extraction optics, mass spectrometer and detectors [1,2]

- SPM - SIMS : Cameca NanoSIMS 50 with integrated AFM/SPM [3-5]

In order to reach good detection limits when probing very small voxels in imaging applications, the ionization probability of the sputtered atoms and molecules needs to be maximized. When using primary ion species such as Ga (as is the case on the integrated TEM - SIMS instrument) or noble gases (HIM - SIMS instrument), the intrinsic yields are low compared to the ones found in conventional SIMS. However, the yields may be drastically increased by using reactive gas flooding during analysis, namely $\mathrm{O}_{2}$ flooding for positive secondary ions and $\mathrm{Cs}$ flooding for negative secondary ions [1,2]. Our results show that both negative and positive ion yields obtained with $\mathrm{Ga}^{+}$ bombardment are increased by up to 4 orders of magnitude when using such reactive gas flooding. This optimization of secondary ion yields leads to detection limits varying from $10^{-3}$ to $10^{-6}$ for a lateral resolution between $10 \mathrm{~nm}$ and $100 \mathrm{~nm}$ (figure 2). 
Different possibilities arise from this in-situ instrument combination. A first approach consists of imaging the sample by SIMS to localize hot spots (e.g. a high concentration of a given element or a given isotope of interest), and then zooming onto this hot spot by TEM/HIM/SPM to identify the feature corresponding to this hot spot. A second approach consists of starting by TEM/HIM/SPM to identify zones or features of interest, and then to determine the chemical composition (or the isotopic ratios) of these features or zones by SIMS (see example in figure 3 for SPM - SIMS). It is important to note that ex-situ multi-technique combinations do not allow the same performances as such an approach is hampered by several limitations, including precise re-localization of analyzed zones after transferring the sample between the standalone instruments and artifacts due to surface oxidation and surface reorganization during sample transfer between the instruments.

The results are very encouraging and the prospects of performing SIMS in combination with TEM, HIM and SPM are very interesting. The combination of high-resolution microscopy and highsensitivity chemical mapping on a single instrument represents a new level of correlative microscopy.

\section{References}

[1] T. Wirtz et al., Appl. Phys. Lett. 101 (2012) 041601

[2] L. Pillatsch et al., Appl. Surf. Sci. 282 (2013) 908

[3] T. Wirtz et al., Surf Interface Anal. 45 (1) (2013) 513-516

[4] T. Wirtz et al., Rev. Sci. Instrum. 83 (2012) 063702

[5] C. L. Nguyen et al., Appl. Surf. Sci. 265 (2013) 489-494

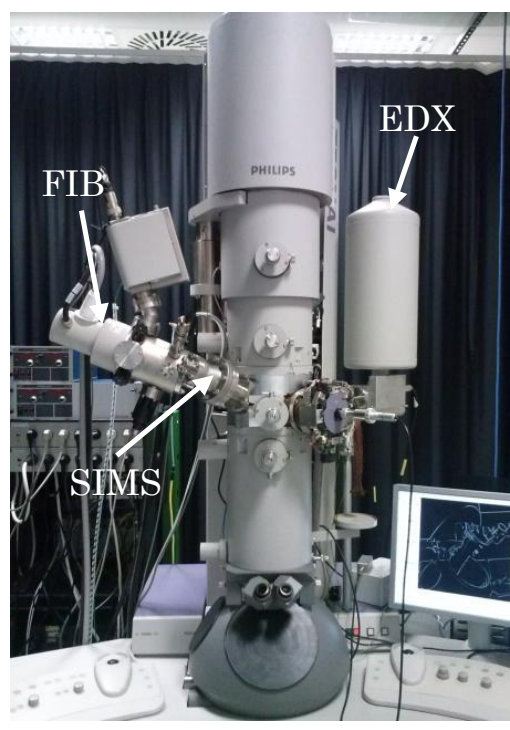

Figure 1: Prototype of a combined TEM-SIMS instrument: modified Tecnai F20 equipped with a $\mathrm{Ga}^{+}$gun and dedicated SIMS column

Figure 3: Combined SIMS-SPM 3D reconstruction of a nickel-based superalloy: $\mathrm{Al}$ distribution (left), $\mathrm{Cr}$ distribution (right)

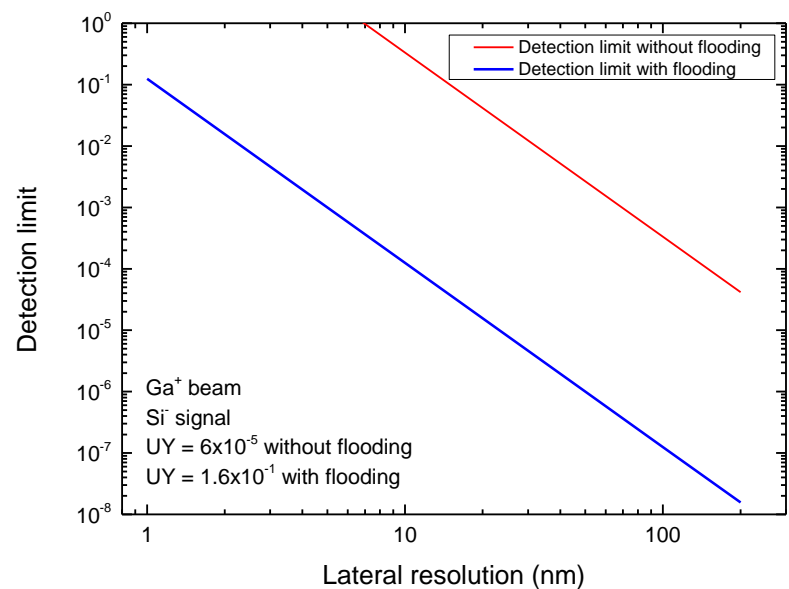

Figure 2: Detection limit using a $\mathrm{Ga}^{+} \mathrm{FIB}$ with and without $\mathrm{Cs}^{0}$ flooding vs. minimum feature size: example for the detection of $\mathrm{Si}^{-}$.

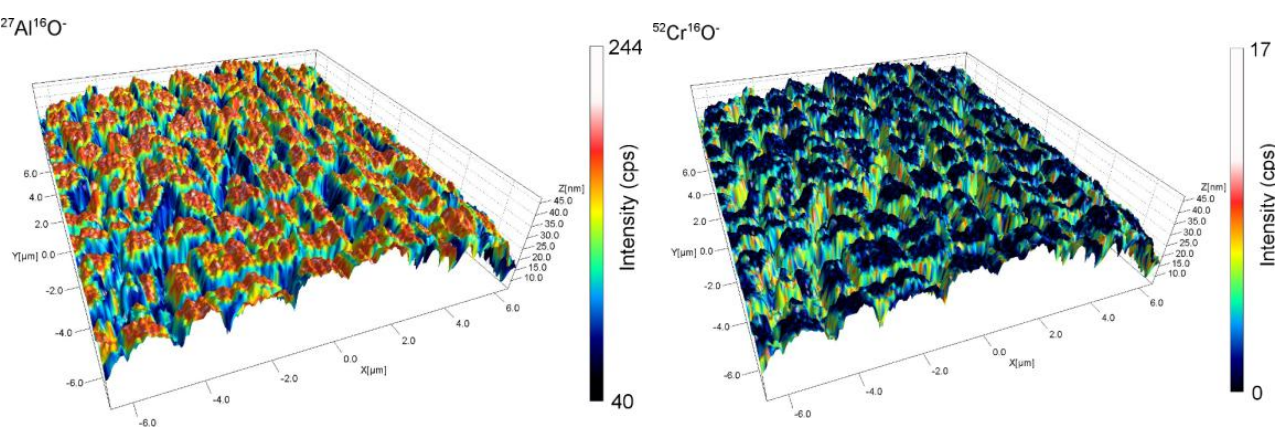

